\title{
Evolution of the plasma sheet electron pitch angle distribution by whistler-mode chorus waves in non-dipole magnetic fields
}

\author{
Q. Ma, B. Ni, X. Tao, and R. M. Thorne \\ Department of Atmospheric and Oceanic Sciences, UCLA, Los Angeles, CA, USA
}

Correspondence to: Q. Ma (qianlima@ atmos.ucla.edu)

Received: 16 November 2011 - Revised: 18 January 2012 - Accepted: 6 February 2012 - Published: 27 April 2012

\begin{abstract}
We present a detailed numerical study on the effects of a non-dipole magnetic field on the Earth's plasma sheet electron distribution and its implication for diffuse auroral precipitation. Use of the modified bounce-averaged Fokker-Planck equation developed in the companion paper by Ni et al. (2012) for 2-D non-dipole magnetic fields suggests that we can adopt a numerical scheme similar to that used for a dipole field, but should evaluate bounce-averaged diffusion coefficients and bounce period related terms in nondipole magnetic fields. Focusing on nightside whistler-mode chorus waves at $L=6$, and using various Dungey magnetic models, we calculate and compare of the bounce-averaged diffusion coefficients in each case. Using the Alternative Direction Implicit (ADI) scheme to numerically solve the 2-D Fokker-Planck diffusion equation, we demonstrate that chorus driven resonant scattering causes plasma sheet electrons to be scattered much faster into loss cone in a non-dipole field than a dipole. The electrons subject to such scattering extends to lower energies and higher equatorial pitch angles when the southward interplanetary magnetic field (IMF) increases in the Dungey magnetic model. Furthermore, we find that changes in the diffusion coefficients are the dominant factor responsible for variations in the modeled temporal evolution of plasma sheet electron distribution. Our study demonstrates that the effects of realistic ambient magnetic fields need to be incorporated into both the evaluation of resonant diffusion coefficients and the calculation of FokkerPlanck diffusion equation to understand quantitatively the evolution of plasma sheet electron distribution and the occurrence of diffuse aurora, in particular at $L>5$ during geomagnetically disturbed periods when the ambient magnetic field considerably deviates from a magnetic dipole.
\end{abstract}

Keywords. Magnetospheric physics (Auroral phenomena; Energetic particles, precipitating) - Space plasma physics (Wave-particle interactions)

\section{Introduction}

The precipitation of low energy plasma sheet electrons is the principal cause of the Earth's diffuse aurora, which is not visually impressive but considerably modifies the ionospheric properties (Eather and Mende, 1971). Electrons diffuse into the loss cone, and thus precipitate into the upper atmosphere due to resonant interactions with the plasma waves in the magnetosphere (e.g., Lyons et al., 1972; Inan et al., 1992). These processes can be modeled using a FokkerPlanck diffusion equation to evaluate the temporal evolution of the electron phase space density (PSD) distribution (Albert, 2004, 2005).

Recently, it has been shown that the most intense nightside diffuse auroral scattering is mainly due to chorus waves, which can lead to the formation of electron pancake distribution at energies below a few keV (e.g., Thorne et al., 2010; Tao et al., 2011; Ni et al., 2011a,b). Most previous studies on the scattering of plasma sheet electrons (e.g., Su et al., 2009; Tao et al., 2011; Horne and Thorne, 2000; Horne et al., 2003; Johnstone et al., 1993; Thorne, 2010) and radiation belt relativistic electrons (e.g., Shprits et al., 2008; Horne et al., 2005; Summers et al., 1998, 2009; Thorne et al., 2005, 2007, 2010) have adopted a dipole field, yet it is known that the Earth's magnetic field is not a perfect dipole, especially at high Lshells or under geomagnetically disturbed conditions. Solar wind disturbance can cause significant changes in the Earth's magnetic field (Baker, 2000). Even at solar quiet times, the dipole field is only a first order approximation. Waveparticle resonant interaction processes can be significantly different when the ambient magnetic field changes (Orlova and Shprits, 2010), which will consequently affect the quantification of magnetospheric electron dynamics (Kennel and Engelmann, 1966). More specifically, for the Earth's diffuse aurora, bounce-averaged diffusion coefficients that critically determine the evolution of electron PSD will be different 
between the use of a magnetic dipole and more realistic nondipole magnetic field models (Ni et al., 2011c).

In the companion paper (Ni et al., 2012), we demonstrate that for 2-D non-dipole magnetic field models it is reasonable to use a bounce-averaged Fokker-Planck diffusion equation similar to that for PSD evolution in a dipole field, but with modified bounce period related terms and bounce-averaged diffusion coefficients. In the present study we choose the nightside Dungey magnetic field model at $L=6$ to simulate the influence of a southward IMF, and focus on the effects of a non-dipole magnetic field on the Earth's diffuse auroral scattering due to upper band chorus (UBC) and lower band chorus (LBC). Section 2 gives a brief description of the generalized formalism of bounce-averaged diffusion coefficients and Fokker-Planck diffusion equation in 2-D magnetic fields. Comparisons of bounce-averaged diffusion coefficients in dipole and Dungey models are shown in Sect. 3. In Sect. 4 we present our modeling of the evolution of plasma sheet electron pitch angle distribution by chorus waves in dipole and non-dipole magnetic fields using the ADI scheme. We discuss implications of the above results in Sect. 5.

\section{Bounce averaged diffusion coefficients and Fokker- Planck diffusion equations in 2-D magnetic fields}

Resonant wave-particle interactions in the Earth's magnetosphere are generally described by quasi-linear diffusion theory (e.g., Albert, 2004). The equations for resonant particle diffusion in pitch angle and energy were first developed by Lyons (1974a,b). The bounce averaged Fokker-Planck equation that describes evolution of phase space density $f$, using any 2-D magnetic field $\boldsymbol{B}=\boldsymbol{B}(\lambda)$ at fixed $L$ is given as (e.g., Schulz, 1976; Schulz and Chen, 1995; Summers, 2005; Ni et al., 2012):

$$
\begin{aligned}
\frac{\partial f}{\partial t}= & \frac{1}{S\left(\alpha_{\mathrm{eq}}\right) \sin \alpha_{\mathrm{eq}} \cos \alpha_{\mathrm{eq}}} \frac{\partial}{\partial \alpha_{\mathrm{eq}}} \\
& \left(S\left(\alpha_{\mathrm{eq}}\right) \sin \alpha_{\mathrm{eq}} \cos \alpha_{\mathrm{eq}}\left\langle D_{\alpha_{\mathrm{eq}} \alpha_{\mathrm{eq}}}\right\rangle \frac{\partial f}{\partial \alpha_{\mathrm{eq}}}\right) \\
+ & \frac{1}{S\left(\alpha_{\mathrm{eq}}\right) \sin \alpha_{\mathrm{eq}} \cos \alpha_{\mathrm{eq}}} \frac{\partial}{\partial \alpha_{\mathrm{eq}}} \\
& \left(S\left(\alpha_{\mathrm{eq}}\right) \sin \alpha_{\mathrm{eq}} \cos \alpha_{\mathrm{eq}}\left\langle D_{\alpha_{\mathrm{eq}} p}\right\rangle \frac{\partial f}{\partial p}\right) \\
+ & \frac{1}{p^{2}} \frac{\partial}{\partial p}\left(p^{2}\left\langle D_{p \alpha_{\mathrm{eq}}}\right\rangle \frac{\partial f}{\partial \alpha_{\mathrm{eq}}}\right)+\frac{1}{p^{2}} \frac{\partial}{\partial p}\left(p^{2}\left\langle D_{p p}\right\rangle \frac{\partial f}{\partial p}\right)(1)
\end{aligned}
$$

Here $p$ is particle momentum, $\alpha_{\mathrm{eq}}$ is equatorial pitch angle, and $S\left(\alpha_{\mathrm{eq}}\right)$ is the bounce period related term. In a dipole field, $S\left(\alpha_{\text {eq }}\right)$ can be approximated by $S\left(\alpha_{\mathrm{eq}}\right)=1.38-$ $0.32 \sin \alpha_{\mathrm{eq}}-0.32 \sqrt{\sin \alpha_{\mathrm{eq}}}$ (e.g., Lenchek et al., 1961; Orlova and Shprits, 2011). In general, $S\left(\alpha_{\mathrm{eq}}\right)$ is given as:

$S\left(\alpha_{\mathrm{eq}}\right)=\int_{\lambda_{\mathrm{m}, \mathrm{s}}}^{\lambda_{\mathrm{m}, \mathrm{n}}} \frac{1}{\cos \alpha} \sqrt{r^{2}+\left(\frac{\partial r}{\partial \lambda}\right)^{2}} d \lambda$, where $r, \lambda$, and $\alpha$ denote radial distance, local magnetic latitude and pitch angle, and the subscripts " $m, s$ " and " $m, n$ " denote mirror points on the Southern and Northern Hemisphere, respectively. In the Fokker-Planck Eq. (1), the parameters $\left\langle D_{\alpha_{\mathrm{eq}} \alpha_{\mathrm{eq}}}\right\rangle,\left\langle D_{p p}\right\rangle$ and $\left\langle D_{\alpha_{\mathrm{eq}} p}\right\rangle=\left\langle D_{p \alpha_{\mathrm{eq}}}\right\rangle$ denote bounce-averaged diffusion coefficients in pitch angle, energy, and mixed terms respectively, which are determined by (e.g., Glauert and Horne, 2005; Summers et al., 2007a,b; Ni et al., 2011c):

$$
\begin{aligned}
\left\langle D_{\alpha_{\mathrm{eq}} \alpha_{\mathrm{eq}}}\right\rangle & =\frac{1}{S\left(\alpha_{\mathrm{eq}}\right)} \int_{\lambda_{\mathrm{m}, \mathrm{s}}}^{\lambda_{\mathrm{m}, \mathrm{n}}} \frac{D_{\alpha \alpha}}{\cos \alpha}\left(\frac{\tan \alpha_{\mathrm{eq}}}{\tan \alpha}\right)^{2} \sqrt{r^{2}+\left(\frac{\partial r}{\partial \lambda}\right)^{2}} d \lambda, \\
\left\langle D_{\alpha_{\mathrm{eq}} p}\right\rangle & =\frac{1}{S\left(\alpha_{\mathrm{eq}}\right)} \int_{\lambda_{\mathrm{m}, \mathrm{s}}}^{\lambda_{\mathrm{m}, \mathrm{s}}} \frac{D_{\alpha p}}{\cos \alpha} \frac{\tan \alpha_{\mathrm{eq}}}{\tan \alpha} \sqrt{r^{2}+\left(\frac{\partial r}{\partial \lambda}\right)^{2}} d \lambda, \\
\left\langle D_{p p}\right\rangle & =\frac{1}{S\left(\alpha_{\mathrm{eq}}\right)} \int_{\lambda_{\mathrm{m}, \mathrm{s}}}^{\lambda_{\mathrm{m}, \mathrm{n}}} \frac{D_{p p}}{\cos \alpha} \sqrt{r^{2}+\left(\frac{\partial r}{\partial \lambda}\right)^{2}} d \lambda,
\end{aligned}
$$

where $D_{\alpha \alpha}, D_{p p}$ and $D_{\alpha p}=D_{p \alpha}$ denote local diffusion coefficients in pitch angle, energy, and mixed terms, respectively. These equations are similar to that in a dipole field (e.g., Lyons and Williams, 1984) except that the bounce time related term $S\left(\alpha_{\mathrm{eq}}\right)$ and bounce-averaged diffusion coefficients need to be calculated in the adopted magnetic field model, which suggests it feasible to use the developed numerical schemes for Fokker-Planck diffusion simulations using a dipole field (e.g., Tao et al., 2008; Xiao et al., 2009).

The Doppler-shifted resonant condition for resonant interactions between electrons and plasma waves is:

$\omega-k_{\|} v_{\|}=n \Omega_{\mathrm{e}} / \gamma$,

where $\omega$ is wave frequency, $k_{\|}$is wave number parallel to the ambient magnetic field, $v_{\|}$is parallel velocity, $n$ is the resonant harmonic order, $\Omega_{\mathrm{e}}$ is electron gyro-frequency, and $\gamma$ is the relativistic factor. When the background magnetic field model changes, the range of electron energy and pitch angle where resonance can occur will change accordingly (e.g., Orlova and Shprits, 2010; Ni et al., 2011c); as shown later, this consequently affects the diffusion coefficients and the evolution of the electron PSD distribution.

\section{Electron resonant diffusion in non-dipole magnetic fields}

\subsection{Dungey magnetic fields and adopted chorus wave model}

The first order approximation to the Earth's magnetic field is a dipole field. However, the Earth's magnetic field is always disturbed and compressed by the ambient solar wind media (Dungey, 1963). The Dungey magnetic model adds a uniform z-component magnetic field $\boldsymbol{B}_{\mathrm{Z}}$ in the Dipole field to simulate the effects of southward IMF: 
Table 1. Parameters of nightside chorus waves at $L=6$ based on CRRES data.

\begin{tabular}{ccccccccccc}
\hline \multirow{2}{*}{ Wave } & Latitude & $B_{\mathrm{w}}(p T)$ & $\overline{f_{\mathrm{m}}}=\frac{f_{\mathrm{m}}}{f_{\mathrm{ce}}}$ & $\overline{f_{\mathrm{lc}}}=\frac{f_{\mathrm{cc}}}{f_{\mathrm{ce}}}$ & $\overline{f_{\mathrm{uc}}}=\frac{f_{\mathrm{uc}}}{f_{\mathrm{ce}}}$ & $\overline{\delta f}=\frac{\delta f}{f_{\mathrm{ce}}}$ & $\theta_{\mathrm{m}}$ & $\theta_{\mathrm{lc}}$ & $\theta_{\mathrm{uc}}$ & $\delta \theta$ \\
\hline LBC & $|\lambda| \leq 5^{\circ}$ & 35.605 & 0.359 & 0.05 & 0.5 & 0.081 & $0^{\circ}$ & $0^{\circ}$ & $58^{\circ}$ & $30^{\circ}$ \\
& $5^{\circ}<|\lambda| \leq 10^{\circ}$ & 73.479 & 0.307 & 0.05 & 0.5 & 0.091 & $20^{\circ}$ & $0^{\circ}$ & $58^{\circ}$ & $30^{\circ}$ \\
& $10^{\circ}<|\lambda| \leq 15^{\circ}$ & 18.336 & 0.234 & 0.05 & 0.5 & 0.113 & $40^{\circ}$ & $0^{\circ}$ & $58^{\circ}$ & $30^{\circ}$ \\
\hline \multirow{2}{*}{ UBC } & $|\lambda| \leq 5^{\circ}$ & 24.864 & 0.582 & 0.5 & 0.7 & 0.060 & $0^{\circ}$ & $0^{\circ}$ & $44^{\circ}$ & $30^{\circ}$ \\
& $5^{\circ}<|\lambda| \leq 10^{\circ}$ & 6.840 & 0.557 & 0.5 & 0.7 & 0.045 & $30^{\circ}$ & $0^{\circ}$ & $44^{\circ}$ & $30^{\circ}$ \\
\hline
\end{tabular}
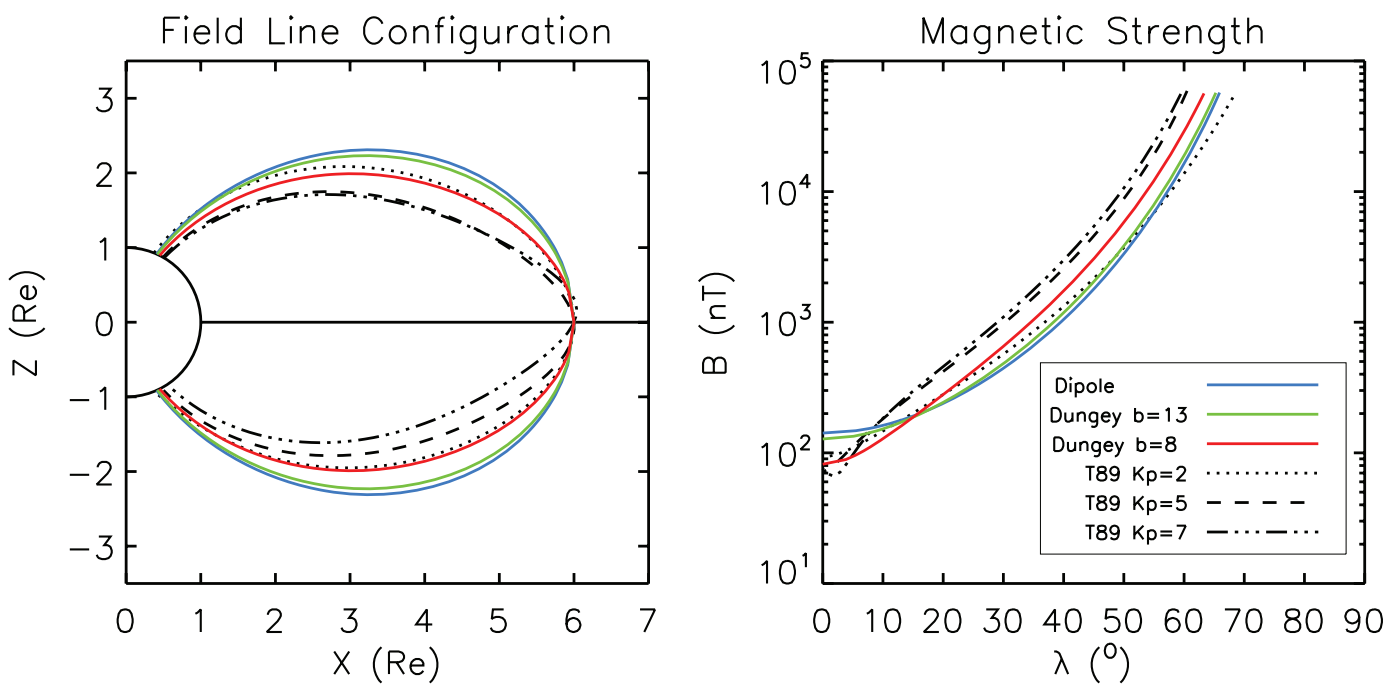

Fig. 1. Comparison of field line configuration (left) and magnetic strength (right) for different magnetic field models at $L=6$. Solid curves are the results from the Dungey magnetic field model when $b$ is 8 (red), 13 (green) and infinity (blue); black dotted, dashed, and dash-dotted curves are results from T89 model when Kp is 2 (quiet), 5 (moderate), and 7 (active), respectively.

$B_{r}=-\left(\frac{2 M}{r^{3}}+B_{\mathrm{z}}\right) \sin \lambda$,

$B_{\lambda}=\left(\frac{2 M}{r^{3}}-B_{\mathrm{z}}\right) \cos \lambda$,

where $M$ is the Earth's dipole magnetic moment. This field model simplifies the Euler potentials description of the Earth's magnetic field at nightside (Kabin et al., 2007). We use $b=\left(M / B_{\mathrm{Z}}\right)^{1 / 3}$ in units of Earth radii as a proxy of the disturbance. The Dungey magnetic model approaches the dipole field when $b$ goes to infinity.

We focus on the resonant wave-particle interaction at $L=$ 6 and magnetic local time (MLT) $=0$. The Dungey magnetic field configuration and magnetic field strength at $L=6$ when $b$ is 8,13 and $\infty$ (dipole) are shown in Fig. 1. For a comparison with the Tsyganenko global empirical magnetic field model we show results for the Tsyganenko 89 (T89) magnetic model (Tsyganenko, 1989) under geomagnetically quiet $(K p=2)$, moderate $(K p=5)$, and active $(K p=7)$ conditions. The southward IMF stretches the Earth's dipole field on the nightside, leading to a decrease in the magnitude of magnetic field strength at lower magnetic latitudes and an increase at higher latitude. Consequently, the resonance condition for wave-particle interaction is considerably affected when we adopt the Dungey magnetic field model compared to a dipole. Based upon the comparison with the T89 results, the Dungey magnetic field model with $b=8$ is more realistic than a dipole field, and gives a reasonable represent of the field distortion.

The parameters of nightside LBC and UBC waves (e.g., Meredith et al., 2001, 2009) at $L=6$ are adopted on the basis of averaged CRRES wave observations (e.g., Ni et al., 2011a,b) under moderately disturbed conditions. Nightside LBC has a latitude distribution $|\lambda|<15^{\circ}$ and frequencies between $0.05 f_{\text {ce }}$ and $0.5 f_{\text {ce }}$, where $f_{\text {ce }}$ is the electron gyrofrequency at equator; Nightside UBC has a latitude distribution $|\lambda|<10^{\circ}$ and frequencies between $0.5 f_{\text {ce }}$ and $0.7 f_{\text {ce }}$. The waves are assumed to have a Gaussian frequency distribution given by (e.g., Glauert and Horne, 2005; Horne et al., 2005):

$B^{2}(\omega)= \begin{cases}A^{2} e^{-\left[\left(\omega-\omega_{\mathrm{m}}\right) / \delta \omega\right]^{2}} & \omega_{\mathrm{lc}} \leq \omega \leq \omega_{\mathrm{uc}} \\ 0 & \text { otherwise }\end{cases}$ 

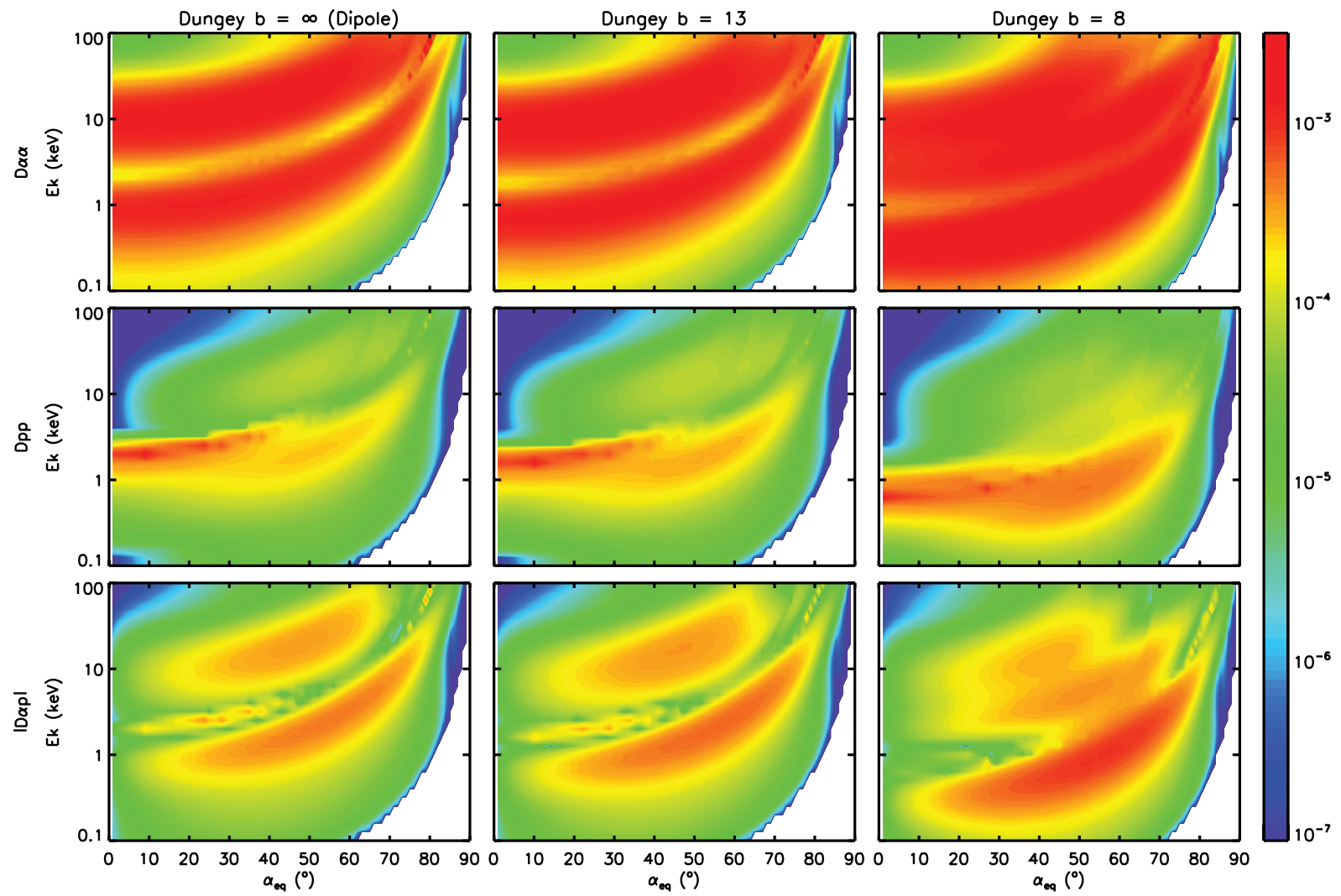

Fig. 2. Bounce-averaged pitch angle diffusion (top), energy diffusion (middle), and mixed diffusion (bottom) coefficients (in units of $s^{-1}$ ) corresponding to use of the Dungey magnetic models with $b=\infty$ (dipole), 13 , and 8 at the equatorial crossing of $L=6$.

where $A$ is a normalized constant given by:

$A^{2}=\frac{\left|B_{\mathrm{w}}\right|^{2}}{\delta \omega} \frac{2}{\sqrt{\pi}}\left[\operatorname{erf}\left(\frac{\omega_{\mathrm{m}}-\omega_{\mathrm{lc}}}{\delta \omega}\right)+\operatorname{erf}\left(\frac{\omega_{\mathrm{uc}}-\omega_{\mathrm{m}}}{\delta \omega}\right)\right]^{-1}$,

and $B_{\mathrm{w}}$ is the wave amplitude. The wave normal angle distribution $g(\theta)$ is also assumed to be Gaussian which can be described as:

$g(X)= \begin{cases}e^{-\left[\left(X-X_{\mathrm{m}}\right) / \delta X\right]^{2}} & X_{\mathrm{lc}} \leq X \leq X_{\mathrm{uc}}, \\ 0 & \text { otherwise, }\end{cases}$

where $X=\tan \theta$. In the three equations above, " $\delta$ " means the bandwidth, subscript " $m$ " means the peak, and subscripts "uc" and "lc" denote the upper cutoff and lower cutoff, respectively. The detailed information of wave amplitude $B_{\mathrm{w}}$, frequency $f$ (normalized by $f_{\text {ce }}$ ) and normal angle $\theta$ are shown in Table 1.

\subsection{Bounce averaged diffusion coefficients}

We use the Full Diffusion Code (FDC) (e.g., Ni et al., 2008; Shprits and Ni, 2009) to calculate the bounce-averaged diffusion coefficients. The bounce-averaged pitch angle, energy, and mixed diffusion coefficients at $L=6$ are shown in Fig. 2 as a function of equatorial pitch angle $\alpha_{\mathrm{eq}}$ and kinetic energy $E_{\mathrm{k}}$ for the three magnetic field models. The two-band frequency structure of chorus leads to characteristic features in diffusion coefficients especially for $D_{\alpha_{\mathrm{eq}} \alpha_{\mathrm{eq}}}$. At lower $\alpha_{\mathrm{eq}}$ the diffusion above a few $\mathrm{keV}$ is mainly caused by LBC while the diffusion below a few $\mathrm{keV}$ is caused by UBC, producing a relatively narrow diffusion gap around a few keV. For the Dungey models ( $b=\infty$ corresponding to a dipole field), as $b$ decreases, resonant diffusion extends to lower energies and higher equatorial pitch angles, tending to diminish the gap between LBC and UBC scattering rates. Clearly, bounceaveraged diffusion coefficients largely depend on the adopted magnetic field model, which is consistent with the results of Orlova and Shprits (2010) and Ni et al. (2011c).

The diffusion coefficients at fixed energies of $300 \mathrm{eV}$, $1 \mathrm{keV}, 3 \mathrm{keV}$ and $10 \mathrm{keV}$ are shown in Fig. 3. Bounceaveraged diffusion coefficients increase for hundreds of $\mathrm{eV}$ electrons when the Earth's magnetic field becomes more stretched. For $1 \mathrm{keV}$ and $3 \mathrm{keV}$ electrons changes in magnetic field can produce larger or smaller scattering rates at lower $\alpha_{\text {eq }}$ compared to the results for a dipole field $(b=\infty)$, depending on the changes in the resonant wave frequencies and the latitudinal extent of resonant interaction. In 

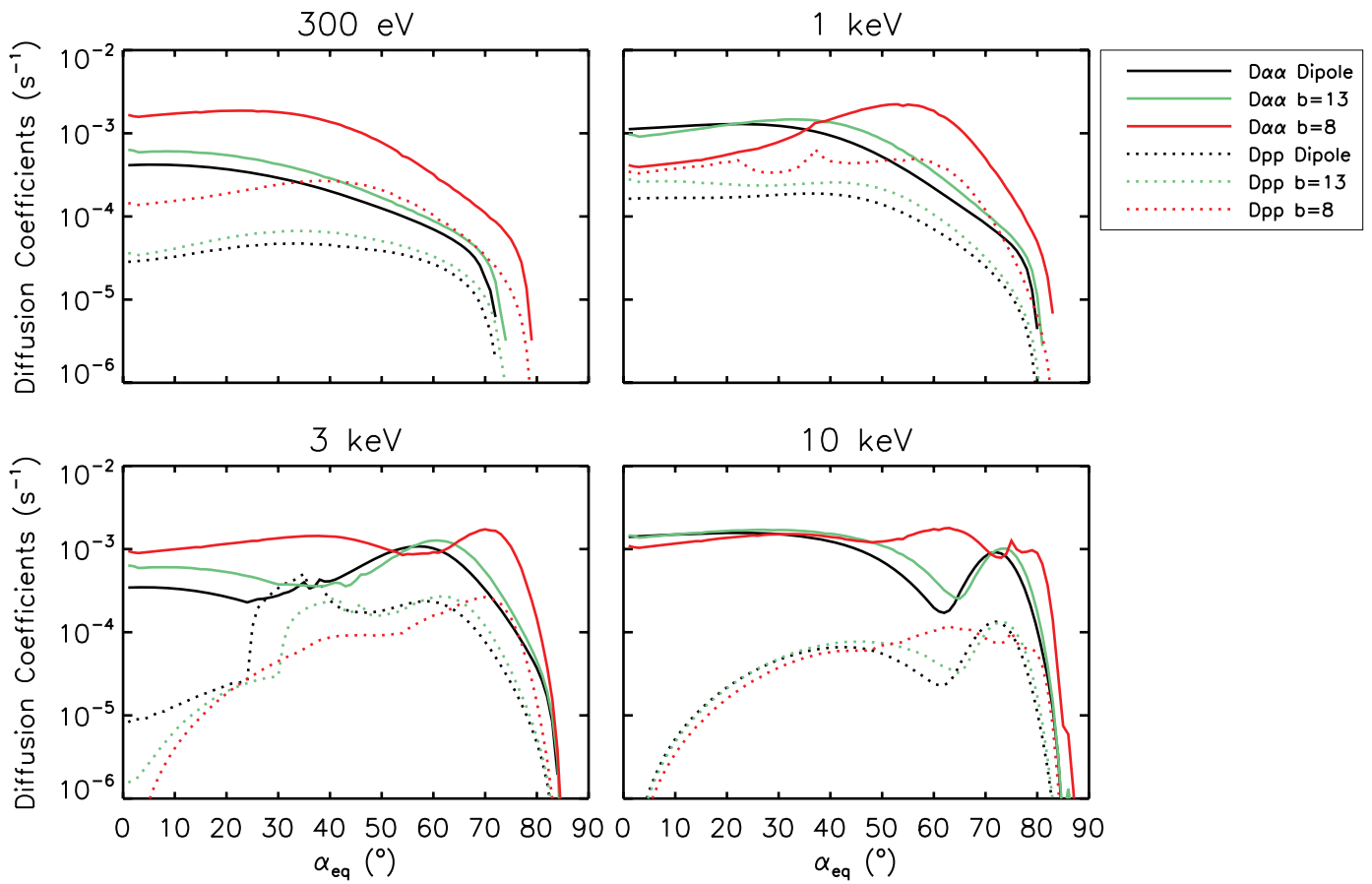

Fig. 3. Bounce-averaged pitch angle diffusion coefficients (solid curves) and energy diffusion coefficients (dotted curves) obtained using Dungey magnetic field models with $b=\infty$ (black), 13 (green) and 8 (red) at $L=6$ and MLT=00:00 for four fixed electron energies of $300 \mathrm{eV}, 1 \mathrm{keV}, 3 \mathrm{keV}$, and $10 \mathrm{keV}$.

contrast, at higher equatorial pitch angle $\left(\alpha_{\mathrm{eq}}>60^{\circ}\right)$, scattering rates are higher in a non-dipole fields. For $10 \mathrm{keV}$ electrons, changes in the diffusion coefficients is relatively small. The redistributions of diffusion coefficients over electron kinetic energy and equatorial pitch angle have significant effects on the evolution of the electron PSD pitch angle distribution, and help identify the waves effects on the particles (e.g., Chen and Schulz, 2001a,b).

\section{Fokker-Planck diffusion simulations in non-dipole fields}

\subsection{Modeled evolution of plasma sheet electron PSD pitch angle distribution}

Compared to the case of using a dipole field, the solutions of the bounce-averaged 2-D Fokker-Planck diffusion equation in non-dipole fields require the evaluation of the bounce period related term $S\left(\alpha_{\mathrm{eq}}\right)$, and the bounce-averaged diffusion coefficients corresponding to the adopted magnetic field model. Polynomial expansion based on $\sqrt{\sin \alpha_{\mathrm{eq}}}$ (e.g., Orlova and Shprits, 2011; Schulz and Lanzerotti, 1974) is used to simulate the bounce period related term in Eq. (2), which gives a relative error of $10^{-3}$. The results of $S\left(\alpha_{\text {eq }}\right)$ for different $b$ values in the Dungey magnetic models show that the normalized bounce time becomes shorter when $b$ varies from infinity (dipole) to 8 , which leads to higher diffusion coefficients and faster diffusion process for the $b=8$ case compared with a dipole or $b=13$ in the Dungey magnetic models.

We can use the numerical schemes developed for a dipole model to calculate the evolution of electron PSD in nondipole fields. A number of numerical methods (e.g., Shprits et al., 2009; Subbotin et al., 2010; Albert and Young, 2005) have been developed for modeling PSD evolution in FokkerPlanck diffusion simulations. In this study we choose the ADI scheme since it is easy to code and computationally efficient (e.g., Xiao et al., 2009; Su et al., 2010b). The Time History of Events and Macroscale Interactions during Substorms (THEMIS) mission (Angelopoulos, 2008) provides observations of electron PSD from a few $\mathrm{eV}$ to $1 \mathrm{MeV}$, and we adopt the nightside data near $L=6$, following Tao et al. (2011), as our initial condition for numerical calculations of PSD evolution, and focus on the energy range from $100 \mathrm{eV}$ to $100 \mathrm{keV}$ which contributes most to the diffuse aurora.

The modeled evolution of plasma sheet electron PSD due to nightside chorus scattering for up to $2 \mathrm{~h}$ is shown in Fig. 4 as a function of equatorial pitch angle and kinetic energy for the three Dungey models. It is clear that electrons are lost rapidly in the energy band between $100 \mathrm{eV}$ to about $20 \mathrm{keV}$ by the combined scattering of LBC and UBC. As the Earth's magnetic field becomes more stretched $(b=8)$, the diffusion in this energy range becomes even faster. This is consistent with the changes in the bounce-averaged diffusion 

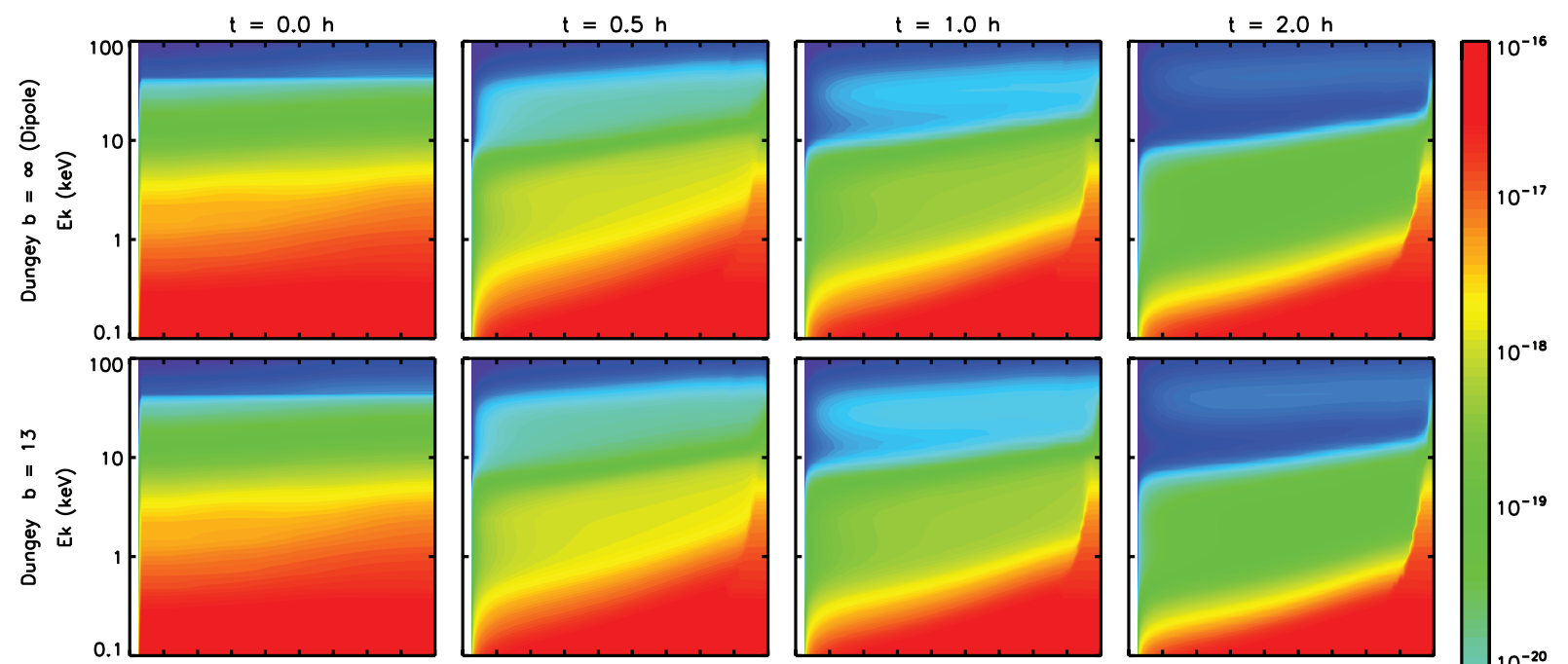

$10^{-18}$
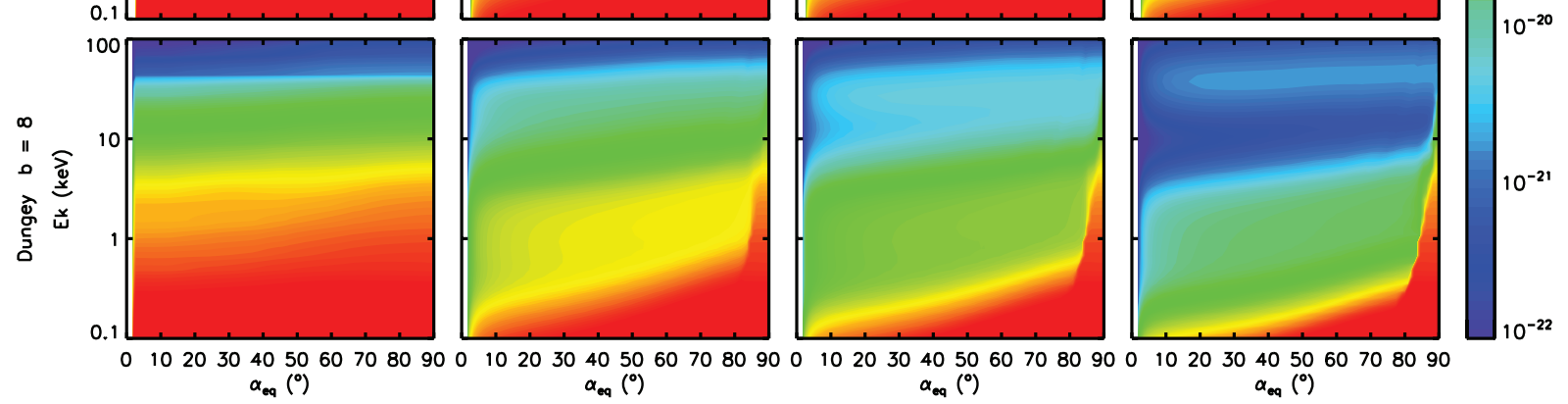

Fig. 4. Evolution of electron PSD (in units of $\mathrm{s}^{3} \mathrm{~m}^{-6}$ ) as a function of equatorial pitch angle and kinetic energy due to nightside chorus at $L=6$ from initial condition (column 1) to $t=0.5 \mathrm{~h}$ (column 2), $t=1.0 \mathrm{~h}$ (column 3 ) and up to $t=2.0 \mathrm{~h}$ (column 4 ) in the Dungey magnetic models with $b=\infty$ (dipole), 13 and 8 .

coefficients that for the more distorted field ( $b=8$ case) wave-particle interaction expands and shifts to lower energy and higher equatorial pitch angle band compared with the dipole field.

In order to study the PSD evolution for this energy band in more detail, Fig. 5 shows the evolution of electron PSD pitch angle distribution at fixed energies from $110 \mathrm{eV}$ to $27.19 \mathrm{keV}$. At energies well below a few $\mathrm{keV}$ ( $2.88 \mathrm{keV}$ in the Dungey $b=\infty$ (dipole) case, $1.78 \mathrm{keV}$ in the Dungey $b=13$ case, and $1.09 \mathrm{keV}$ in the Dungey $b=8$ case), electron loss is dominantly due to UBC scattering and the electron PSD distributions form pancake structures. At energies well above several $\mathrm{keV}(7.59 \mathrm{keV}$ in the Dungey $b=\infty$ (dipole) case, $6.46 \mathrm{keV}$ in the Dungey $b=13$ case, and $2.88 \mathrm{keV}$ in the Dungey $b=8$ case), the electron scattering is mainly caused by LBC at lower equatorial pitch angles and UBC at higher equatorial pitch angle. The combination of rapid pitch angle scattering loss by LBC and energy diffusion by UBC leads to an increase in the PSD distribution anisotropy with time. For the energies between the above two energy bands, the scattering loss is relatively slower. The reduction in electron loss contributes to the formation of a flattened PSD distribution, consistent with Tao et al. (2011).
Comparisons of PSD evolution among the three Dungey fields clearly show that the temporal variation of electron PSD pitch angle distribution is strongly dependent on the adopted magnetic field models. Compared to the results using the dipole field, electron PSD at lower equatorial pitch angle decreases faster in stretched, non-dipole Dungey field as $b$ decreases. Due to the extension of scattering rates to higher $\alpha_{\text {eq }}$, drops in electron PSD also occur over a broader $\alpha_{\text {eq }}$ range (up to higher $\alpha_{\text {eq }}$ ) in non-dipole Dungey fields. In addition, the decrease in electron PSD is much larger for the cases of $b=8$, especially for electrons from $100 \mathrm{eV}$ to $15 \mathrm{keV}$, suggesting a much more pronounced precipitation loss of plasma sheet electrons during disturbed periods.

\subsection{Relative roles of bounce-averaged diffusion coeffi- cients and $S\left(\alpha_{\mathrm{eq}}\right)$}

In order to better understand the factors responsible for the differences in electron PSD evolution introduced by use of different magnetic field models, we perform further 2-D Fokker-Planck diffusion simulations to investigate the relative roles of bounce-averaged diffusion coefficients and bounce period related term $S\left(\alpha_{\mathrm{eq}}\right)$, since these are the only two terms that are changed when we switch from the dipole 

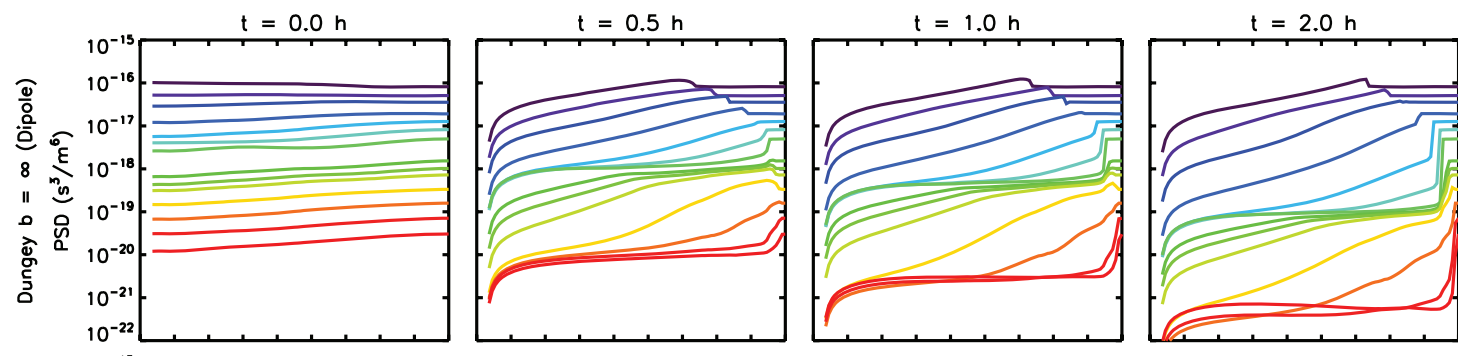

Ek (keV)

27.19

19.81

14.40

10.46

7.59
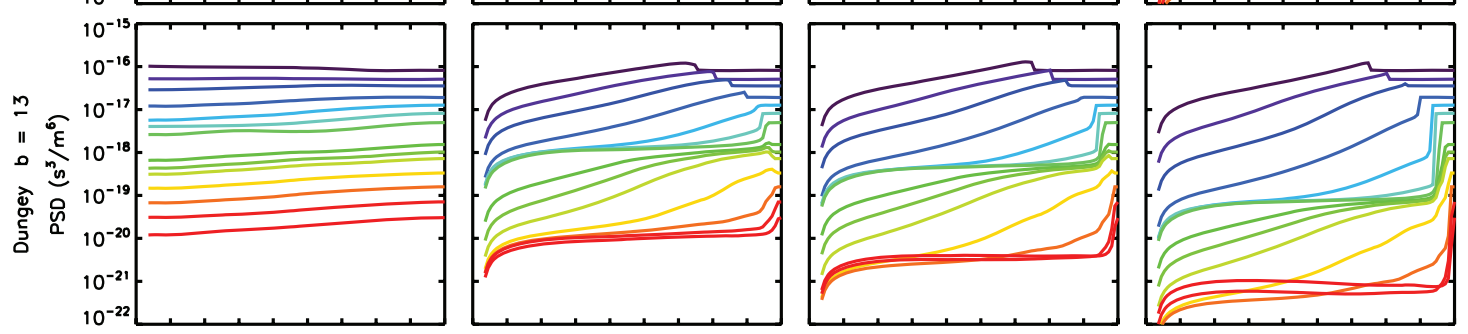

6.46

5.50

2.88

1.78

1.09
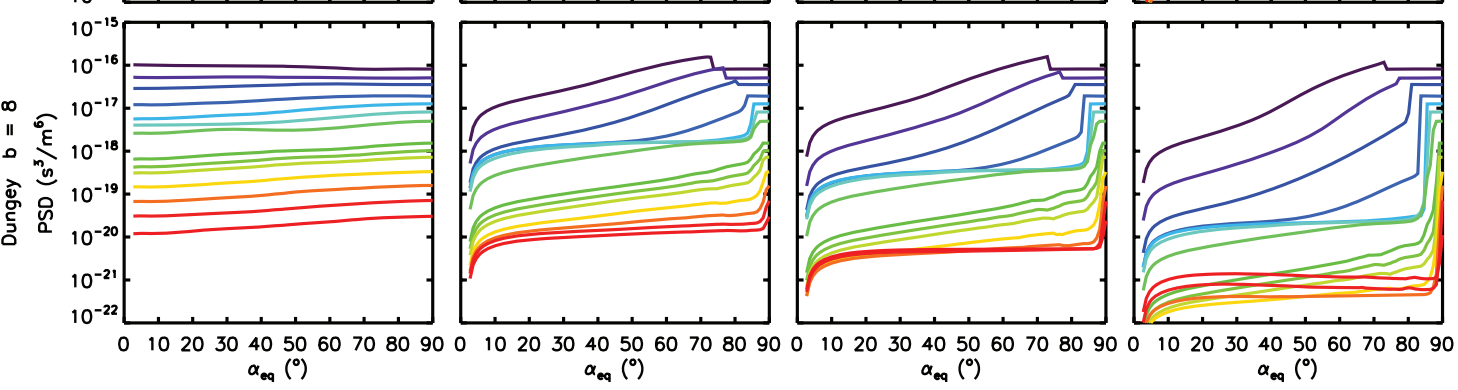

0.57

0.30

0.18

0.11

Fig. 5. Evolution of PSD $\left(\mathrm{s}^{3} \mathrm{~m}^{-6}\right)$ as a function of equatorial pitch angle at selected fixed energies due to nightside chorus at $L=6$ from initial condition (column 1) to $t=0.5 \mathrm{~h}$ (column 2), $t=1.0 \mathrm{~h}$ (column 3 ) and up to $t=2.0 \mathrm{~h}$ (column 4 ) in the Dungey magnetic models with $b=\infty$ (dipole), 13 and 8 .

model to the Dungey models. The results shown in Fig. 6 indicate that when we adopt the Dungey model with $b=8$ and only change $S\left(\alpha_{\mathrm{eq}}\right)$, the difference compared to simulation in a dipole field becomes more pronounced at higher energy of a few $\mathrm{keV}$. However, changes in the bounce-averaged diffusion coefficients have a strong effect at both lower and higher energy bands where the loss by UBC and LBC waves are most prominent. Overall, changes in bounce-averaged diffusion coefficients are more effective for influencing the PSD evolution within different magnetic field models.

\section{Conclusion and discussion}

We numerically solved the bounce-averaged diffusion equation in non-dipole 2-D magnetic models to understand the effects of different magnetic models on electron PSD distribution in Earth's diffuse aurora zone. Bounce-averaged diffusion coefficients in different magnetic models were computed, and the resulting PSD evolution was calculated by ADI scheme. Specifically, the electron diffusion by LBC and UBC waves (Bortnik and Thorne, 2007), which contributes to the Earth's diffuse aurora most, was studied in the Dungey field models with $b=\infty$ (dipole), 13 , and 8 .
Our results show a similar behavior with the previous studies performed in dipole fields (e.g., Tao et al., 2011; Ni et al., 2008), but the rate of precipitation is quantitatively different when using the Dungey $b=8$ magnetic model. We find that the bounce-averaged diffusion coefficients are generally stronger and shifts to lower energy and higher equatorial pitch angle bands for the Dungey $b=8$ model, which is the most distorted case. Correspondingly, electron PSD distribution is also lost faster with diffusion extending to lower energy and higher equatorial pitch angle bands, which suggests that the non-dipole component and disturbance in Earth's magnetic fields can cause quantitative changes in the diffusion process of plasma sheet electrons. Considering that the Dungey model with $b=8$ is the most realistic when compared with T89 magnetic field results, ignoring the southward magnetic field component and using a pure dipole field will significantly underestimate the PSD loss in earth's diffusive aurora zone, especially at energies below $15 \mathrm{keV}$.

Further modeling will require more realistic nonsymmetric 3-D magnetic models to model the electron PSD evolution (e.g., Albert et al., 2009; Fok et al., 2008; Jordanova et al., 2010; Su et al., 2010a; Xiao et al., 2010). The magnetic models used here are confined at MLT $=0$, while the Earth's magnetic field configuration varies significantly 

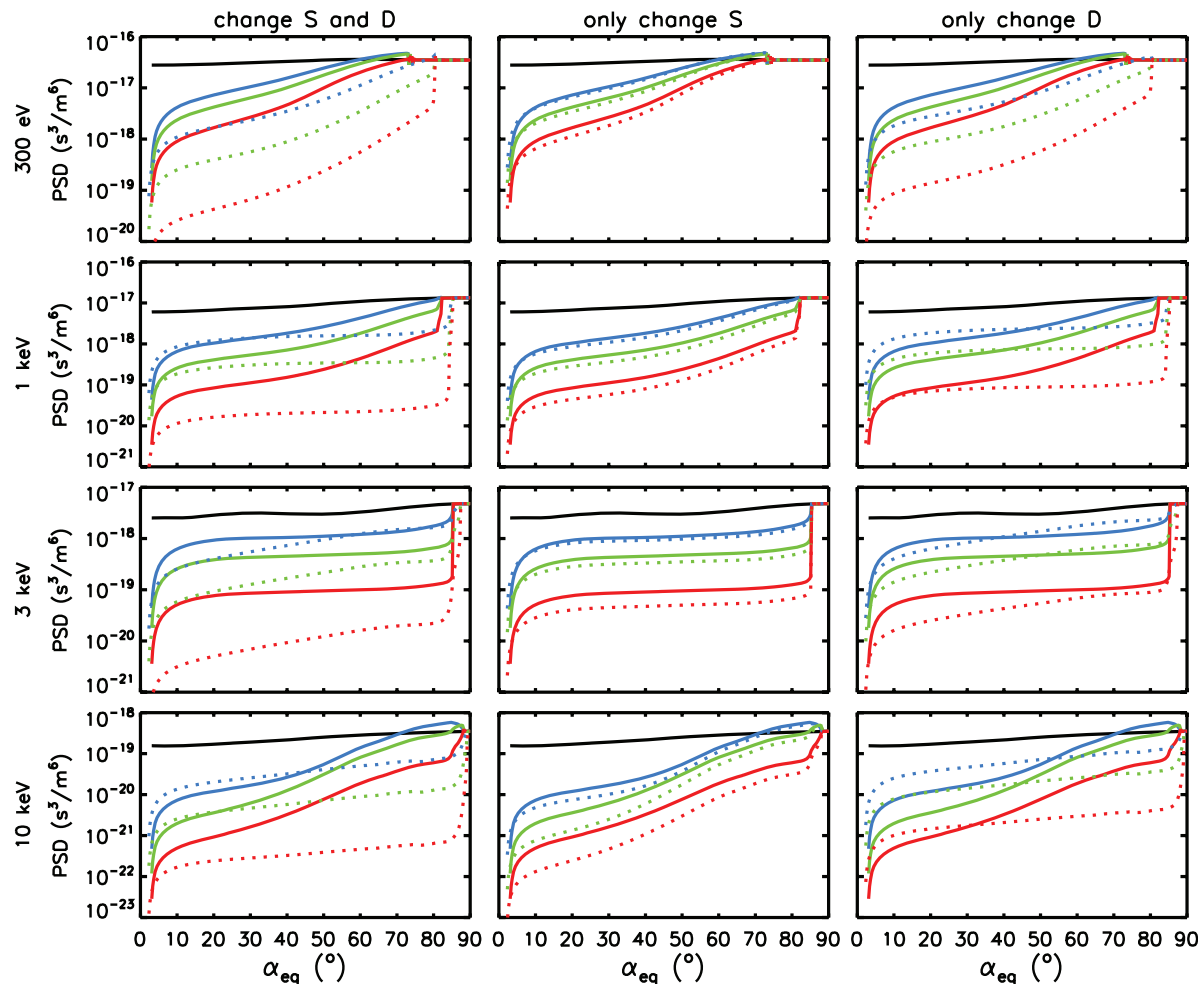

only change D
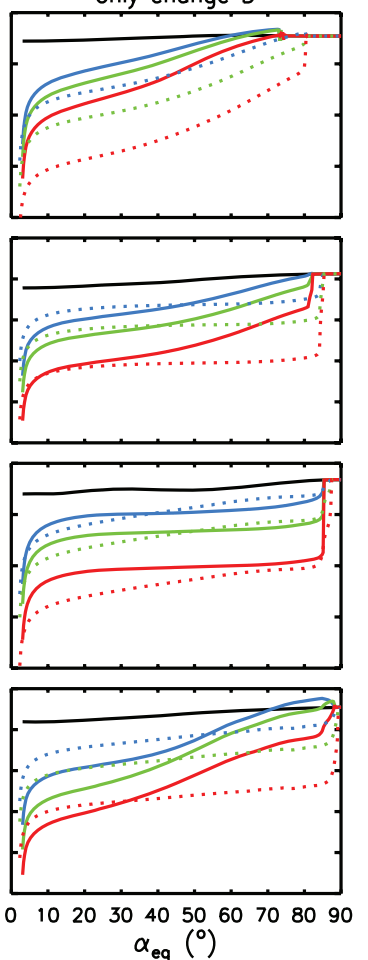

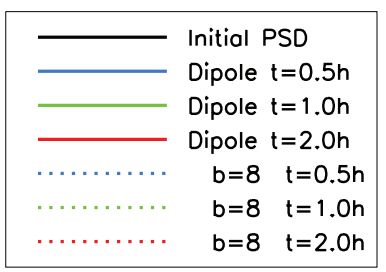

Fig. 6. Comparison of the effects of bounce period related term $S\left(\alpha_{\mathrm{eq}}\right)$ and bounce-averaged diffusion coefficients $(D)$ on electron PSD $\left(\mathrm{s}^{3} \mathrm{~m}^{-6}\right.$ ) evolution by using different magnetic field models: dipole (solid) and the Dungey model $b=8$ (dotted). Results are given for four specified energies of $300 \mathrm{eV}, 1 \mathrm{keV}, 3 \mathrm{keV}$ and $10 \mathrm{keV}$ after four time scales of PSD evolution: $t=0.0 \mathrm{~h}$ (black), $t=0.5 \mathrm{~h}$ (blue), $t=1.0 \mathrm{~h}$ (green) and $t=2.0 \mathrm{~h}(\mathrm{red})$.

with MLT. The adoption of non-dipole magnetic field is important in multi-dimensional Fokker-Planck diffusion simulations for both low energy plasma sheet electrons and radiation belt relativistic electrons. This work shows the study of non-dipole effects on the wave-particle interaction in the Earth's diffuse aurora, and suggests that the choice of different magnetic models could have a significant influence on the plasma sheet electron PSD evolution.

Acknowledgements. This research was supported in part by NSF grant ATM-0802843. The authors would like to thank Michael Schulz and Yuri Shprits for useful discussions.

Topical Editor R. Nakamura thanks J. Albert and M. Chen for their help in evaluating this paper.

\section{References}

Albert, J. M.: Using quasi-linear diffusion to model acceleration and loss from wave-particle interactions, Space Weather, 2, S09S03, doi:10.1029/2004SW000069, 2004.

Albert, J. M.: Evaluation of quasi-linear diffusion coefficients for whistler mode waves in a plasma with arbitrary density ratio, J. Geophys. Res., 110, A03218, doi:10.1029/2004JA010844, 2005.
Albert, J. M. and Young, S. L.: Multidimensional quasi-linear diffusion of radiation belt electrons, Geophys. Res. Lett., 32, L14110, doi:10.1029/2005GL023191, 2005.

Albert, J. M., Meredith, N. P., and Horne, R. B.: Three-dimensional diffusion simulation of outer radiation belt electrons during the 9 October 1990 magnetic storm, J. Geophys. Res., 114, A09214, doi:10.1029/2009JA014336, 2009.

Angelopoulos, V.: The THEMIS Mission, Space Sci. Rev., 141, 534, doi:10.1007/s11214-008-9336-1, 2008.

Baker, D. N.: Effects of the Sun on the Earth's environment, J. Atmos. Solar-Terr. Phys., 62, 1669-1681, doi:10.1016/S13646826(00)00119-X, 2000.

Bortnik, J. and Thorne, R. M.: The dual role of ELF/VLF chorus waves in the acceleration and precipitation of radiation belt electrons, J. Atmos. Solar-Terr. Phys., 69, 378-386, 2007.

Chen, M. and Schulz, M.: Simulations of storm time diffuse aurora with plasmasheet electrons in strong pitch angle diffusion, J. Geophys. Res., 106, 1873-1886, 2001a.

Chen, M. and Schulz, M.: Simulations of diffuse aurora with plasma sheet electrons in pitch angle diffusion less than everywhere strong, J. Geophys. Res., 106, 28949-28966, 2001 b.

Dungey, J. W.: Interactions of solar plasma with the geomagnetic field, Planet Space Sci., 10, 233-237, doi:10.1016/00320633(63)90020-5, 1963.

Eather, R. H. and Mende, S. B.: Airborne Observations of Auroral Precipitation Patterns, J. Geophys. Res., 76, 1746-1755, 
doi:10.1029/JA076i007p01746, 1971.

Fok, M., Horne, R. B., Meredith, N. P., and Glauert, S. A.: Radiation Belt Environment model: Application to space weather nowcasting, J. Geophys. Res., 113, A03S08, doi:10.1029/2007JA012558, 2008.

Glauert, S. A. and Horne, R. B.: Calculation of pitch-angle and energy diffusion coefficients with the PADIE code, J. Geophys. Res., 110, A04206, doi:10.1029/2004JA010851, 2005.

Horne, R. B. and Thorne, R. M.: Electron pitch angle diffusion by electrostatic electron cyclotron harmonic waves: The origin of pancake distributions, J. Geophys. Res., 105, 5391-5402, 2000.

Horne, R. B., Thorne, R. M., Meredith, N. P., and Anderson, R. R.: Diffuse auroral electron scattering by electron cyclotron harmonic and whistler mode waves during an isolated substorm, J. Geophys. Res., 108, 1290, doi:10.1029/2002JA009736, 2003.

Horne, R. B., Thorne, R. M., Glauert, S. A., Albert, J. M., Meredith, N. P., and Anderson, R. R.: Timescale for radiation belt electron acceleration by whistler mode chorus waves, J. Geophys. Res., 110, A03225, doi:10.1029/2004JA010811, 2005.

Inan, U. S., Chiu, Y. T., and Davidson, G. T.: Whistler-mode chorus and morningside aurorae, Geophys. Res. Lett., 19, 653-656, doi:10.1029/92GL00402, 1992.

Johnstone, A., Walton, D., Liu, R., and Hardy, D.: Pitch angle diffusion of low-energy electrons by whistler mode waves, J. Geophys. Res., 98, 5959-5967, 1993.

Jordanova, V. K., Zaharia, S., and Welling, D. T.: Comparative study of ring current development using empirical, dipolar, and self-consistent magnetic field simulations, J. Geophys. Res., 115, A00J11, doi:10.1029/2010JA015671, 2010.

Kabin, K., Rankin, R., Mann, I. R., Degeling, A. W., and Marchand, R.: Polarization properties of standing shear Alfvén waves in non-axisymmetric background magnetic fields, Ann. Geophys., 25, 815-822, doi:10.5194/angeo-25-815-2007, 2007.

Kennel, C. F. and Engelmann, F.: Velocity Space Diffusion from Weak Plasma Turbulence in a Magnetic Field, The Physics of Fluids, 9, 2377-2388, 1966.

Lenchek, A., Singer, S., and Wentworth, R.: Geomagnetically Trapped Electrons from Cosmic Ray Albedo Neutrons, J. Geophys. Res., 66, 4027-4046, doi:10.1029/JZ066i012p04027, 1961.

Lyons, L. R.: General relations for resonant particle diffusion in pitch angle and energy, J. Plasma Physics, 12, 45-49, 1974a.

Lyons, L. R.: Pitch angle and energy diffusion coefficients from resonant interactions with ion-cyclotron and whistler waves, J. Plasma Physics, 12, 417-432, 1974b.

Lyons, L. R. and Williams, D. J.: Quantitative aspects of magnetospheric physics, p. 140, Springer, New York, 1984.

Lyons, L. R., Thorne, R. M., and Kennel, C. F.: Pitch-angle diffusion of radiation belt electrons within the plasmasphere, J. Geophys. Res., 77, 3455-3474, doi:10.1029/JA077i019p03455, 1972.

Meredith, N., Horne, R., and Anderson, R.: Substorm dependence of chorus amplitudes: Implications for the acceleration of electrons to relativistic energies, J. Geophys. Res., 106, 1316513178, 2001.

Meredith, N. P., Horne, R. B., Thorne, R. M., and Anderson, R. R.: Survey of upper band chorus and ECH waves: Implications for the diffuse aurora, J. Geophys. Res., 114, A07218, doi:10.1029/2009JA014230, 2009.
Ni, B., Thorne, R. M., Shprits, Y. Y., and Bortnik, J.: Resonant scattering of plasma sheet electrons by whistler-mode chorus: Contribution to diffuse auroral precipitation, Geophys. Res. Lett., 35, L11106, doi:10.1029/2008GL034032, 2008.

Ni, B., Thorne, R. M., Horne, R. B., Meredith, N. P., Shprits, Y. Y., Chen, L., and Li, W.: Resonant scattering of plasma sheet electrons leading to diffuse auroral precipitation: 1. Evaluation for electrostatic electron cyclotron harmonic waves, J. Geophys. Res., 116, A04218, doi:10.1029/2010JA016232, 2011a.

Ni, B., Thorne, R. M., Meredith, N. P., Horne, R. B., and Shprits, Y. Y.: Resonant scattering of plasma sheet electrons leading to diffuse auroral precipitation: 2. Evaluation for whistler mode chorus waves, J. Geophys. Res., 116, A04219, doi:10.1029/2010JA016233, 2011 b.

Ni, B., Thorne, R. M., Shprits, Y. Y., Orlova, K. G., and Meredith, N. P.: Chorus-driven resonant scattering of diffuse auroral electrons in nondipolar magnetic fields, J. Geophys. Res., 116, A06225, doi:10.1029/2011JA016453, 2011c.

Ni, B., Thorne, R. M., and Ma, Q.: Bounce-averaged Fokker-Planck diffusion equation in non-dipolar magnetic fields with application to the Dungey magnetosphere, Ann. Geophys., 30, 733-750, doi:10.5194/angeo-30-733-2012, 2012.

Orlova, K. G. and Shprits, Y. Y.: Dependence of pitch-angle scattering rates and loss timescales on the magnetic field model, Geophys. Res. Lett., 37, L05105, doi:10.1029/2009GL041639, 2010.

Orlova, K. G. and Shprits, Y. Y.: On the bounce-averaging of scattering rates and the calculation of bounce period, Phys. Plasmas, 18, 092904, doi:10.1063/1.3638137, 2011.

Schulz, M.: Particle motion and diffusion in geometric mirrors, Phys. Fluids, 19, 1835-1836, 1976.

Schulz, M. and Chen, M.: Bounce-averaged Hamiltonian for charged particles in an axisymmetric but nondipolar model magnetosphere, J. Geophys. Res., 100, 5627-5635, 1995.

Schulz, M. and Lanzerotti, L. J.: Particle Diffusion in the Radiation Belts, Phys. Chem. Space, 7, 215 pp., Springer, New York, 1974

Shprits, Y. Y. and Ni, B.: Dependence of the quasi-linear scattering rates on the wave normal distribution of chorus waves, J. Geophys. Res., 114, A11205, doi:10.1029/2009JA014223, 2009.

Shprits, Y. Y., Subbotin, D. A., Meredish, N. P., and Elkington, S. R.: Review of modeling of losses and sources of relativistic electrons in the outer radiation belt II: Local acceleration and loss, J. Atmos. Solar-Terr. Phys., 70, 1694-1713, doi:10.1016/j.jastp.2008.06.014, 2008.

Shprits, Y. Y., Subbotin, D., and Ni, B.: Evolution of electron fluxes in the outer radiation belt computed with the VERB code, J. Geophys. Res., 114, A11209, doi:10.1029/2008JA013784, 2009.

Su, Z., Zheng, H., and Wang, S.: Evolution of electron pitch angle distribution due to interactions with whistler mode chorus following substorn injections, J. Geophys. Res., 114, A08202, doi:10.1029/2009JA014269, 2009.

Su, Z., Xiao, F., Zheng, H., and Wang, S.: STEERB: A three-dimensional code for storm-time evolution of electron radiation belt, J. Geophys. Res., 115, A09208, doi:10.1029/2009JA015210, 2010a.

Su, Z., Zheng, H., and Wang, S.: Three-dimensional simulation of energetic outer zone electron dynamics due to wave-particle interaction and azimuthal advection, J. Geophys. Res., 115, A06203, doi:10.1029/2009JA014980, 2010b.

Subbotin, D., Shprits, Y., and Ni, B.: Three dimensional VERB 
radiation belt simulations including mixed diffusion, J. Geophys. Res., 115, A03205, doi:10.1029/2009JA015070, 2010.

Summers, D.: Quasi-linear diffusion coefficients for field-aligned electromagnetic waves with applications to the magnetosphere, J. Geophys. Res., 110, A08213, doi:10.1029/2005JA011159, 2005.

Summers, D., Thorne, R. M., and Xiao, F.: Relativistic theory of wave-particle resonant diffusion with application to electron acceleration in the magnetosphere, J. Geophys. Res., 103, $20487-$ 20500, 1998.

Summers, D., Ni, B., and Meredith, N. P.: Timescales for radiation belt electron acceleration and loss due to resonant waveparticle interactions: 1. Theory, J. Geophys. Res., 112, A04206, doi:10.1029/2006JA011801, 2007a.

Summers, D., Ni, B., and Meredith, N. P.: Timescales for radiation belt electron acceleration and loss due to resonant wave-particle interactions: 2. Evaluation for VLF chorus, ELF hiss, and electromagnetic ion cyclotron waves, J. Geophys. Res., 112, A04207, doi:10.1029/2006JA011993, 2007b.

Summers, D., Tang, R., and Thorne, R. M.: Limit on stably trapped particle fluxes in planetary magnetospheres, J. Geophys. Res., 114, A10210, doi:10.1029/2009JA014428, 2009.

Tao, X., Chan, A. A., Albert, J. M., and Miller, J. A.: Stochastic modeling of multidimensional diffusion in the radiation belts, J. Geophys. Res., 113, A07212, doi:10.1029/2007JA012985, 2008.

Tao, X., Thorne, R. M., Li, W., Ni, B., Meredith, N. P., and Horne, R. B.: Evolution of electron pitch angle distributions following injection from the plasma sheet, J. Geophys. Res., 116, A04229, doi:10.1029/2010JA016245, 2011.
Thorne, R. M.: Radiation belt dynamics: The importance of wave-particle interactions, Geophys. Res. Lett., 37, L22107, doi:10.1029/2010GL044990, 2010.

Thorne, R. M., O’Brien, T. P., Shprits, Y. Y., Summers, D., and Horne, R. B.: Timescale for $\mathrm{MeV}$ electron microburst loss during geomagnetic storms, J. Geophys. Res., 110, A09202, doi:10.1029/2004JA010882, 2005.

Thorne, R. M., Shprits, Y. Y., Meredith, N. P., Horne, R. B., Li, W., and Lyons, L. R.: Refilling of the slot region between the inner and outer electron radiation belts during geomagnetic storms, J. Geophys. Res., 112, A06203, doi:10.1029/2006JA012176, 2007.

Thorne, R. M., Ni, B., Tao, X., Horne, R. B., and Meredith, N. P.: Scattering by chorus waves as the dominant cause of diffuse auroral precipitation, Nature, 467, 943-946, doi:10.1038/nature09467, 2010.

Tsyganenko, N. A.: A magnetospheric magnetic field model with a warped tail current sheet, Planet. Space Sci., 37, 5-20, doi:10.1016/0032-0633(89)90066-4, 1989.

Xiao, F., Su, Z., Zheng, H., and Wang, S.: Modeling of outer radiation belt electrons by multidimensional diffusion process, J. Geophys. Res., 114, A03201, doi:10.1029/2008JA013580, 2009.

Xiao, F., Su, Z., Zheng, H., and Wang, S.: Three-dimensional simulations of outer radiation belt electron dynamics including cross-diffusion terms, J. Geophys. Res., 115, A05216, doi:10.1029/2009JA014541, 2010. 